\title{
Trend of Anti-SARS-COV-2 Seropositivity in Karachi
}

\author{
Nayab Afzal*, Naila Tariq, Danish Shakeel, Sadia Sultan \\ Department of Laboratory Services, National Medical Center, Karachi, Pakistan.
}

\begin{abstract}
Objective: This study aims to determine the trend of seropositivity of anti-SARS-CoV-2 antibodies (total including IgM, Ig G) in patients presenting at a private hospital in Karachi.

Materials \& Methods: A total of 2500 patientswere included in the study. Informed consent was obtained and a questionnaire including patient's age, gender, symptoms, history of traveling, contact with people who had travelled recently, contact with confirmed/suspected COVID-19 patient at home or work was filled. Samples were analyzed for anti-SARS-CoV-2 antibodies on Roche Cobase601.
\end{abstract}

Results: The mean age of the patients was 41.045 \pm 20.48 years. 674(26.96\%) were reactive, while $1826(73.04 \%)$ were non-reactive. Mostly patients were males $1388(55.52 \%)$. The seroprevalence was higher in males $29.75 \%$ while females showed $23.47 \%$ seroprevalence. Commonest symptoms were found to be fever $(88.73 \%)$ and dry cough $(57.20 \%)$. The frequency of seropositive patients revealed an increasing trend with $20.5 \%$ and $27.8 \%$ in first andlast 15 days of June respectively, $30.7 \%$ and $31.4 \%$ in first andlast 15 days of July 2020 respectively.Only $221(32.79 \%)$ reactive patients reported recent symptoms while $453(67.21 \%)$ patients were completely asymptomatic.Odds Ratio(OR) for participants with recent history of travelling, contact with people who had travelled recently,exposure with COVID-19 patients at home or work was $11.50,3.28,8.45$ and 0.72 respectively.

Conclusion: The seropositivity has shown a definite gradual upward trend over the study period and approximately two thirds of the patients being completely asymptomatic pointing towards the fact that many people are silently exposed to the disease and develop antibodies through their natural immune mechanism.

Keywords: COVID 19, Anti-SARS-CoV-2 antibodies, Trend, Seropositivity, Dry cough, Diarrhea.

\section{INTRODUCTION}

Novel Coronavirus has taken the world by storm with its first cases in Wuhan, China reported in December 2019 [1]. On 11 February 2020, the virus was given the taxonomic nomenclature of "Severe Acute Respiratory Syndrome Coronavirus 2 (SARS-CoV-2)" by the World Health Organization (WHO); causing Coronavirus Disease 2019 (COVID-19) [2]. The highly contagious nature of this disease led to a massive spread across the globe bringing the numbers to a staggering height of 16,298,556 confirmed cases, according to the most recent WHO COVID-19 situation reports (dated July 30, 2020) [3]. As medical personals tried to grasp a hold of the worsening situationthe testing strategieshave evolved massively within a short course of eight months. Initially RT-PCR was the main stay for diagnosis which was mostly offered to symptomatic patients [4] but the world soon realized that the number of asymptomatic patients was far greater than what was previously anticipated leading to gross underestimation of prevalence of COVID-19. These patients were found to be silent carriers of the disease as they did not seek medical help due to no or very mild symptoms leading to rapid community spread of COVID-19 [5]. As more and more cases appeared the picture started to become clearer and scientific efforts were directed towards detection of antibodies against coronavirus for a better understanding of the

*Address correspondence to this author at the Department of Laboratory Services, National Medical Center, Karachi, Pakistan.

Email: nayabfaizan@gmail.com disease demographics and the extent of spread.

Pakistan is the fifth most populous country of the world with Karachi being the largest city that is home to about 16 million people [6]. Pakistan reported its first confirmed case of COVID-19 in the end of February, 2020 in Karachi. In the initial phase of this disease all of the confirmed cases had recent travel history from Iran, Syria and London $[7,8]$ but now most of the cases are due to local dissemination of SARS-CoV-2 [7]. The total number confirmed cases have risen to 528,891 and deaths toll stands at 11,204 as of 22nd January 2021 in Pakistan [9]. In Sindh the number of confirmed cases is close to 239,186 and death toll is 3855 [9]. These numbers of confirmed cases are based on RT-PCR test results, which is expensive and not as widely available. Furthermore, a vast majority (80\%) of COVID-19 patients may remain asymptomatic or develop only mild to moderate symptoms [5] not requiring immediate hospitalization, thus they do not get RT-PCR test done in spite of suffering from COVID-19 serving as a source for infection dissemination [10]. Data restricted to only the confirmed cases do not project the true spread of virus or fatality associated with this disease. Therefore, it can be safely said that these statistics are not representative of the actual disease burden in Pakistani population. A better estimate of the extent of infected asymptomatic/mildly symptomatic population is crucial to plan evidence-based strategies for combating this pandemic. Assessment of seroprevalence of anti-SARS-CoV-2 antibod- 
ies, can greatly help the policy makers to navigate in these turbulent times. Here, we present the seroprevalence of anti-SARS-CoV-2 antibodies in patients visiting outpatient department and emergency of a tertiary care hospital in Karachi for better understanding of current disease burden and development of result oriented strategies in the near future.

\section{MATERIALS AND METHODS}

This cross sectional study was conducted from June 2020 to July 2020 at the Clinical Chemistry section of National Medical Center Laboratory Karachi. The study was approved by ethical committee of the National Medical Centre, Karachi, Pakistan (Ref. No. Nil dated May 30, 2020). A total of 2500 consenting patients who visited the outpatient or emergency department of the hospital were included in this study. Sampling technique used was Non probability consecutive sampling. Study purpose was thoroughly explained to the patients. Questionnaire regarding age, gender, symptoms (dry cough, fever, shortness of breath, sore throat, flu, loss of taste/smell, headaches, body aches, rash, diarrhea and vomiting,travel in past 14 days, contact with people who have travelledin past 14 days, contact with confirmed/suspected COVID-19 patient at home or at work was filled by the patient.

Venous blood samples were collected using serum separator tube (SST). Tubes were immediately centrifuged at $4000 \mathrm{~g}$ for 15 minutes to separate the serum. Anti-SARS-CoV-2 antibodies were detected using fully automated Roche cobas e601 immunoassay analyzer based on Electro-chemiluminescence Immunoassay (ECLIA) technique. The specificity of this test is reported to be $99.81 \%(99.67-99.90 \%)$. The calibration and internal quality control was done according to manufacturer's recommendations.

Data was organized and entered on SPSS version 22.Patients were labeled as reactive (cut off $>1.000$ ) and non-reactive (cut off $<1.000)$. Age was presented as Mean \pm standard deviation. The frequency of seropositive patients in first 2 weeks and last 2 weeks of June and July 2020 were calculated. Frequency of symptoms, history of positive RT PCR and seropositivity in different age groups was noted.We also calculated the odds ratio (OD) of being seropositive for each subset of history of traveling, exposure with people who have travelled, exposure with confirmed/suspected COVID-19 patient at home or at work.

\section{RESULTS}

The mean age of the patients was $41.045 \pm 20.48$ years. Additionally the largest proportion of participants belonged to $19-60$ years age group $(n=1743,67.6 \%)$. Most of the patients were males 1388(55.52\%). $674(26.96 \%)$ were reactive, while $1826(73.04 \%)$ were non-reactive. The seroprevalence in males was found to be $29.75 \%$ as compared to females, who showed $23.47 \%$ seroprevalence, age also had an effect on seroprevalence (Table 1). Only $222(32.79 \%)$ reactive patients reported recent symptoms while $453(67.21 \%)$ patients were completely asymptomatic. Fever was noted to be the commonest symptom followed by dry cough (Table 2). The frequency of seropositive patients revealed an increasing trend with $20.5 \%$ in first 2 weeks of June, $27.8 \%$ in last 15 days of June, $30.7 \%$ in first 2 weeks of July, $31.4 \%$ in last 15 days of July 2020 as depicted in (Fig. 1). Odds Ratio (OR) for participants with recent history of travelling, contact with people who had travelled recently, exposure with COVID-19 patients at home or work was $11.5,3.28,8.45$ and 0.72 respectively (Table 3).

Table 1. Demographics of Participants, Frequency of Anti-SARS CoV-2 Seropositivity according to Gender and Age $(\mathrm{n}=2500)$.

\begin{tabular}{|c|c|c|c|c|c|}
\hline Variables & Options & $\begin{array}{c}\text { No. of } \\
\text { Participants } \\
\text { (n) }\end{array}$ & $\begin{array}{c}\text { Percentage } \\
\mathbf{( \% )}\end{array}$ & $\begin{array}{c}\text { Reactive } \\
\mathbf{( \% )}\end{array}$ & $\begin{array}{c}\text { Non- } \\
\text { Reactive } \\
\mathbf{( \% )}\end{array}$ \\
\hline Gender & Males & 1388 & 55.52 & $413(29.75)$ & $975(70.25)$ \\
& Females & 1112 & 44.48 & $261(23.47)$ & $851(76.53)$ \\
\hline Age & $0-18$ & 273 & 13.7 & $49(17.95)$ & $224(82.05)$ \\
(Years) & $19-60$ & 1743 & 67.6 & $499(28.70)$ & $1244(71.30)$ \\
& $>60$ & 484 & 18.6 & $126(26.04)$ & $358(73.96)$ \\
\hline
\end{tabular}

Table 2. Frequency of Different Symptoms $(n=222)$.

\begin{tabular}{|l|c|}
\hline Symptoms & No. of Participants (\%) \\
\hline Fever & $197(88.73 \%)$ \\
\hline Dry cough & $127(57.20 \%)$ \\
\hline Body aches & $119(53.60 \%)$ \\
\hline Shortness of breath & $82(36.94 \%)$ \\
\hline Headache & $57(25.67 \%)$ \\
\hline Sore throat & $34(15.32 \%)$ \\
\hline Loss of smell & $25(11.26 \%)$ \\
\hline Loss of taste & $19(8.5 \%)$ \\
\hline Diarrhea & $13(5.85 \%)$ \\
\hline Flu & $12(5.40 \%)$ \\
\hline Vomiting & $8(3.6 \%)$ \\
\hline Rash & $6(2.75)$ \\
\hline
\end{tabular}


Table 3. Odds Ratio for Patients with History of Travelling, Contact with People who have Travelled in Past 15 Days Covid-19 Exposure at Home and COVID-19 Exposure at Work.

\begin{tabular}{|c|c|c|c|c|c|}
\hline \multirow[t]{2}{*}{ Patients with History of } & \multicolumn{2}{|c|}{ Anti Sars CoV 2 Test } & \multirow[t]{2}{*}{ Odds Ratio } & \multirow[t]{2}{*}{$95 \% \mathrm{CI}$} & \multirow[t]{2}{*}{ p-Value } \\
\hline & Reactive\% & Non-reactive $\%$ & & & \\
\hline Travelling & $55(79.7)$ & $14(20.3)$ & \multirow{2}{*}{11.50} & \multirow{2}{*}{$6.35-20.83$} & \multirow{2}{*}{$<0.001$} \\
\hline No travelling & $619(25.5)$ & $1813(74.5)$ & & & \\
\hline Contact with people who have travelled & $19(54.3)$ & $16(45.7)$ & \multirow{2}{*}{3.28} & \multirow{2}{*}{$1.67-6.42$} & \multirow{2}{*}{0.0005} \\
\hline No contact with people who have travelled & $655(26.6)$ & $1810(73.4)$ & & & \\
\hline Exposure at home & $30(75)$ & $10(25)$ & \multirow{2}{*}{8.45} & \multirow{2}{*}{$4.11-17.40$} & \multirow{2}{*}{$<0.0001$} \\
\hline No exposure at home & $644(26.2)$ & $1816(73.8)$ & & & \\
\hline Exposure at work & $22(21.4)$ & $81(78.6)$ & \multirow{2}{*}{0.72} & \multirow{2}{*}{$0.44-1.17$} & \multirow{2}{*}{0.19} \\
\hline No exposure at work & $652(27.2)$ & $1745(72.8)$ & & & \\
\hline
\end{tabular}

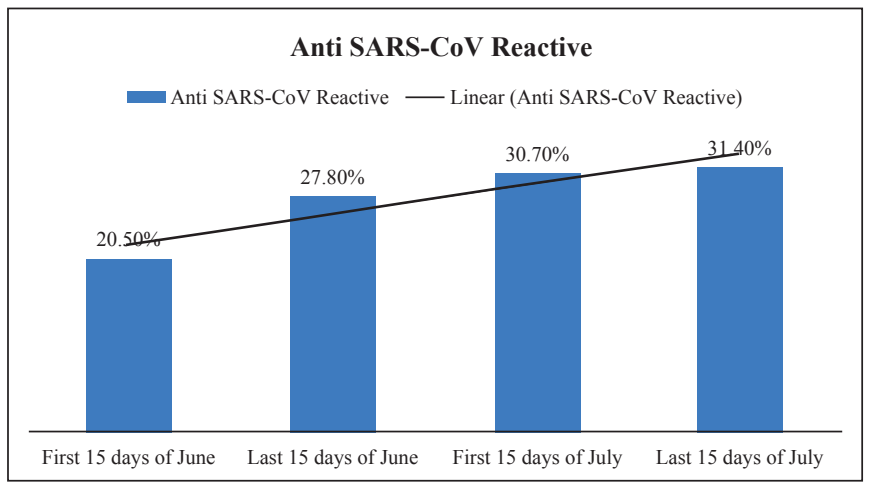

Fig. (1). Trend of Anti SARS CoV-2 Seropositivity in the Months of June and July 2020.

\section{DISCUSSION}

After making its debut in China at the end of last year, COVID-19 reached Pakistan on February 26, 2020. As of 22nd January 2021 the total number confirmed cases is 528,891 and deaths toll stands at 11,204 [9]. In Sindh the number of confirmed cases is close to 239,186 and death toll is 3855 [9]. However, due to easing of lock down imposed by the government of Pakistan and non-compliance of necessary precautionary measures by the public, graph of new reported cases and daily deaths is showing an upward trend.

This study predominantly highlights the Anti SARS CoV-2 seropositivity in the months of June and July 2020 in Karachi, Pakistan. The demographic data of patientsrevealedmore males $(55.52 \%)$ as compared to females $(44.48 \%)$. Another study by Stringhini S, et al. which indicated that $47.6 \%$ of participants were males.11Males were found to have greater frequency of seropositivity (29.75\%) as compared to females (23.47\%). Higher seroprevalence in male population could be due to relatively more social activities of males in our region or stronger immune response than females. However these are simple speculations as no scientific data is available on this subject. On the other hand studies conducted in Switzerland and Spain and reported no gender difference [11, 12].

Maximum percentage of our participants belonged to 19-60 years age group (67.6\%) and children displayed lower seroprevalence (13.7\%) studies from Geneva and Spain reflect a similar pattern $[10,11]$. Whether this reflects a low susceptibility of infection in children or just low pathogen exposure of these children due to closure of schools, day-care units and parks remains to be elucidated.

Evidence collected in this study shows that $67.06 \%$ remained asymptomaticand only $32.93 \%$ developed symptoms. This high prevalence of asymptomatic patients being reactive for Anti SARS CoV-2 could be due to strong immune response or less virulence of the virushowever asymptomatic cases were $28.5 \%$ and $50.5 \%$ in studies conducted by Pollán M, et al. and Wang Y, et al. respectively $[12,13]$. These findings could be due to difference in demographics and immune response of our population.

The patients in our study reported fever as the commonest symptom 197(88.73\%),followed by dry cough 127 (57.20\%) and body aches 119 (53.60\%). Similar findings were noted by Wang Y, et al. [14]. Some patients also presented with gastrointestinal symptoms like diarrhea and vomiting. These observations are in line with reports by Centers for Disease Control and Prevention (CDC) [15]. Some patients presented with unusual symptom like rash 6 (2.7\%).

The frequency of seropositive patients revealed an increasing trend with $20.5 \%$ in first 15 days of June, $27.8 \%$ in last 15 days of June, $30.7 \%$ in first 15 days of July, 31.4\% in last 15 days of July 2020. This observation is particularly interesting considering the fact that total number of active cases in Pakistan had started to show a gradual consistent decline as early as first week of July. The climbing trend for Anti SARS CoV-2 may point towards slow but steady progress towards herd immunity against COVID-19. These particular observations can prove to be massively helpful in understanding the actual disease burden in our population. Similar findings have 
been reported in another study revealing an increase in frequency of seropositive patients over a period of five weeks [10].

Upon further data analysis significant association was observed between anti SARS CoV 2 seropositivity and history of travelling in past 14 days $(\mathrm{OR}=11.50)$, history of contact with people who have travelled in past 14 days $(\mathrm{OR}=3.28)$ and history of exposure to COVID-19 patient at home $(\mathrm{OR}=8.45)$. However, no significant association was found between exposure to COVID-19 at work $(\mathrm{OR}=0.72)$. These results could be explained on basis of a general lack of social distancing measures at home or when travelling and relatively strict use of PPEs at work places due to rules imposed by organizations. Proper use of PPEs, reducing the number of passengers, preflight passenger screening, temperature testing, hand sanitization and improved air conditioning system can help in reduction of risk of exposure to COVID-19 while travelling [16, 17]. Occupational Safety and Health Administration (OSHA) and the US Centers for Disease Control (CDC) have provided special COVID-19 pandemic associated recommendations for employers'. These consist of several controls at the levels of administration, engineering and PPE usage [18-20].

Our study has few limitations. First, it only includes patients who visited the hospital. A survey with a large sample size which includes a wider section of population can reflect more reliable statistical figures regarding the seroprevalence of anti-SARS-CoV-2 antibodies. Furthermore, this study was conducted over the duration of only two months. Extensive analysis of such data over a continued period of time is recommended to get a better understanding of the infection and its associated entities, which can greatly benefit the policy makers in devising possiblestrategies to combat the pandemic. We could not elucidate difference by race, as this question was notaddressed. Additionally the average time for appearance of Anti SARS CoV-2 is 14 days after the onset of symptoms. Samples collected very shortly after patient developed symptoms could cause false negative results, therefore the seroprevalence could be underestimated.

All the physicians should obtain history regarding, mild COVID-19 like symptoms, recent travel and possible disease exposure as COVID-19 is now spreading mainly through local transmission. Rigorous practice of this multipronged approach can help us limit this pandemic in our region.

\section{CONCLUSION}

Our study provides a comprehensive estimate of prevalence of Anti SARS CoV-2 in a vast number of patients covering all age groups. A remarkably increasing trend of Anti SARS CoV-2 seropositivity is noted over the period of this study. Our results highlight the fact that many people are silently exposed to this disease and eventually develop antibodies. This could be potential head start towards development of herd immunity in our population. Such studies also provide essential insight about actual disease burden and immunological state of the population.

\section{LIST OF ABBREVIATIONS}

SARS-CoV-2 Severe Acute Respiratory Syndrome Coronavirus 2.

WHO World Health Organization.

COVID-19 Coronavirus Disease 2019 .

CDC Centers for Disease Control and Prevention.

OSHA Occupational Safety and Health. Administration.

\section{AUTHORS' CONTRIBUTION}

All authors have contributed equally.

\section{FUNDING}

The authors have received no funding for this article.

\section{CONFLICT OF INTEREST}

Declared none.

\section{ACKNOWLEDGEMENTS}

We acknowledge all our patients for their participation in the study.

\section{REFERENCES}

[1] Zhu N, Zhang D, Wang W, et al. A novel coronavirus from patients with pneumonia in China, 2019. N Engl J Med 2020; 382: 727-33. DOI: 10.1056/NEJMoa2001017

[2] Baig AM, Khaleeq A, Ali U, et al. Evidence of the COVID-19 virus targeting the CNS: Tissue distribution, host-virus interaction, and proposed neurotropic mechanisms. ACS Chem Neurosci 2020; 11(7): 995-8. DOI: 10.1021/acschemneuro.0c00122

[3] World Health Organization. Novel coronavirus (COVID-19) situation reports. 2020; Available at: https://www.who.int/ e m ergen cies / dis e a s es / nove 1-coronavirus-2019/situation-reports. Accessed: [May 20, 2020].

[4] Guo YR, Cao QD, Hong ZS, et al. The origin, transmission and clinical therapies on coronavirus disease 2019 (COVID-19) outbreak - an update on the status. Mil Med Res 2020; 7(1): 11. DOI: 10.1186/s40779-020-00240-0

[5] Gao Z, Xu Y, Sun C, et al. A systematic review of asymptomatic infections with COVID-19. J Microbiol Immunol Infect 2020; 54(1): 12-6. DOI: 10.1016/j.jmii.2020.05.001

[6] http://www.worldometers.info/world-population/pakistan-population/ 
[7] Waris A, Atta UK, Ali M, Asmat A, Baset A. COVID-19 outbreak: Current scenario of Pakistan. New Microbes New Infect 2020; 35: 100681. DOI: 10.1016/j.nmni.2020.100681

[8] Javed B, Sarwer A, Soto EB, Mashwani ZU. Is Pakistan on track to have COVID-19 transmission and mortality rates similar to those of Italy, Iran or the USA? Drugs Ther Perspect 2020; 1-5. DOI: 10.1007/s40267-020-00726-W

[9] https://covid.gov.pk/stats/pakistan

[10] Wu Z, McGoogan JM. Characteristics of and important lessons from the coronavirus disease 2019 (COVID-19) outbreak in China: Summary of a report of 72314 cases from the Chinese Center for Disease Control and Prevention. JAMA 2020; 323(13): 1239-42. DOI: 10.1001/jama.2020.2648

[11] Stringhini S, Wisniak A, Piumatti G, et al. Seroprevalence ofanti-SARS-CoV-2 IgG antibodies in Geneva, Switzerland (SEROCoV-POP): A population-based study. Lancet 2020; 396(10247): 131-9. DOI: 10.1016/S0140-6736(20)31304-0

[12] Pollán M, Pérez-Gómez B, Pastor-Barriuso R, et al. Prevalence of SARS-CoV-2in Spain (ENE-COVID): A nationwide, population-based seroepidemiological study. Lancet 2020; 396(10250): 535-44. DOI: 10.1016/S0140-6736(20)32266-2

[13] Wang Y, Kang H, Liu X, Tong Z. Asymptomatic cases with SARS-CoV-2 infection. J Med Virol 2020; 92(9): 1401-3. DOI: $10.1002 / j m v .25990$
[14] Wang Y, Wang Y, Chen Y, Qin Q. Unique epidemiological and clinical features of the emerging 2019 novel coronavirus pneumonia (COVID-19) implicate special control measures. J Med Virol 2020; 92(6): 568-76. DOI: 10.1002/jmv.25748

[15] Centers for Disease Control and Prevention. Symptoms of coronavirus. 2020; Available at: https:/www.cdc.gov/coronavirus/2019-ncov/symptoms-testing/symptoms.html. [Accessed 19 August, 2020].

[16] The Lancet Infectious Diseases. Air travel in the time of COVID-19. Lancet Infect Dis 2020; 20(9): 993. DOI: 10.1016/S1473-3099(20)30647-2

[17] Bielecki M, Patel D, Hinkelbein J, et al. Air travel and COVID-19 prevention in the pandemic and peri-pandemic period: A narrative review. Travel Med Infect Dis 2020; 39: 101915. DOI: $10.1016 /$ j.tmaid.2020.101915

[18] Occupational Safety and Health Administration (OSHA). Guidance on Preparing Workplaces for COVID-19. Washington, DC: US Department of Labor 2020.

[19] https://www.cdc.gov/coronavirus/2019-ncov/communication/guidance.html [Accessed 18 August, 2020].

[20] Zisook RE, Mannot A, Parker J, et al. Assessing and managing the risks of COVID-19 in the workplace: Applying industrial hygiene (IH)/occupational and environmental health and safety (OEHS) frameworks. Toxicol Ind Health 2020; 36(9): 607-618. DOI: 10.1177/0748233720967522 